\title{
Os centros regionais inovativos de tecnologia assistiva do Brasil
}

\author{
The regional innovative centers of assistive technology of Brazil
}

\section{Los centros regionales innovadores de tecnología asistiva de Brasil}

\author{
Marcus Vinícius Albrecht Anversa ${ }^{1}$ \\ Universidade do Estado do Rio de Janeiro, Brasil
}

\begin{abstract}
Resumo
A situação do Brasil, segundo o Censo do IBGE 2010 (BRASIL, 2012), é de 23,9 \% dos brasileiros apresentarem ao menos algum tipo de deficiência que se pode enquadrar como: visual, motora, auditiva ou mental. Destes, cerca de $18,6 \%$ possuem deficiência visual, $7 \%$ motora, $5,1 \%$ auditiva, e 1,4\% mental. A Organização Mundial de Saúde (2011) concluiu que no decorrer da vida humana, em algum momento, ocorrerá algum tipo de deficiência, sendo a mais provável por processo de envelhecimento. Em vista deste quadro, a busca por uma sociedade mais inclusiva se tornou uma das prerrogativas das Políticas Públicas de Ciência, Tecnologia e Inovação Brasileira, principalmente a partir da $4^{a}$ Conferência Nacional de Ciência, Tecnologia e Inovação $\left(4^{\mathrm{a}} \mathrm{CNCTI}\right)$, realizada em 2010. A determinação e estudo dos Centros Regionais Inovativos de Tecnologia Assistiva, portanto, urge como necessidade em fornecer instrumentos para tais políticas.
\end{abstract}

Palavras-chaves: Centros Regionais Inovativos, Patentes, Inovação Tecnológica, Tecnologia Assistiva

\section{Abstract}

According to the IBGE Census 2010 (BRASIL, 2012), the Brazilian situation is $23.9 \%$ of Brazilians have at least some type of disability that can be classified as visual, motor, hearing or mental disabilities. Of these, about $18.6 \%$ have visual impairment, $7 \%$ motor, $5.1 \%$ auditory and $1.4 \%$

1 Doutor em Geografia pela Universidade do Estado do Rio de Janeiro - UERJ. Pesquisador do Grupo de Pesquisa de Novas Tecnologias Voltadas à Produção e Divulgação do Conhecimento em Geografia - UERJ. Analista em Ciência e Tecnologia do MCTIC / Instituto Nacional de Tecnologia - INT. Correio eletrônico: marcus.anversa@int.gov.br

Este artículo corresponde a la ponencia presentada en el 35th Conference of Latin American Geographers realizada en San José, Costa Rica del 20 al 22 de mayo del 2018. 
mental. The World Health Organization (2011) concluded that during human life, at some point, some type of disability will occur, being the most likely due to the aging process. In view of this situation, the search for a more inclusive society has become one of the prerogatives of the Brazilian Science, Technology and Innovation Public Policy, especially since the 4th National Conference on Science, Technology and Innovation (4th CNCTI) held in 2010.The determination and study of the Innovative Regional Centers for Assistive Technology, therefore, urgently needs to provide instruments for such policies.

Keywords: Innovative Regional Centers, Patents, Technological Innovation, Assistive Technology

\section{Resumen}

Según el Censo del IBGE 2010 (BRASIL, 2012), el 23,9\% de los brasileños presentan al menos algún tipo de discapacidad como: visual, motora, auditiva o mental. De ellos, cerca del 18,6\% poseen deficiencia visual, 7\% motora, 5,1\% auditiva y 1,4\% mental. La Organización Mundial de la Salud (2011) concluyó que en el transcurso de la vida humana, en algún momento, ocurrirá algún tipo de deficiencia, siendo la más probable por proceso de envejecimiento. En vista de este panorama la búsqueda por una sociedad más inclusiva se ha convertido en una de las prerrogativas de las Políticas Públicas de Ciencia, Tecnología e Innovación Brasileña, principalmente a partir de la $4^{\text {a }}$ Conferencia Nacional de Ciencia, Tecnología e Innovación (4 ${ }^{\text {a }}$ CNCTI), realizada en 2010. La determinación y estudio de los Centros Regionales Innovadores de Tecnología Asistiva, por lo tanto, urge como necesidad en proveer instrumentos para tales políticas.

Palabras claves: Centros Regionales Innovadores, Patentes, Innovación Tecnológica, Tecnología Asistiva

\section{Introdução}

Tecnologia Assistiva (TA) é uma expressão recente referente a um conceito ainda em pleno processo de construção e sistematização. A utilização dessa tecnologia poderia se dizer que remonta aos primórdios da história da humana, ou até mesmo da pré-história. Qualquer pedaço de pau utilizado como uma bengala improvisada, por exemplo, caracteriza o uso de um recurso de Tecnologia Assistiva.

Como afirma José Eduardo Manzini (2005)

Os recursos de tecnologia assistiva estão muito próximos do nosso dia a dia. Ora eles nos causa impacto devido à tecnologia que apresentam, ora passam quase despercebidos. Para exemplificar, podemos chamar de tecnologia assistiva uma bengala, utilizada por nossos avôs para proporcionar conforto e segurança no momento de caminhar, bem como um aparelho de amplificação utilizado por uma pessoa com surdez moderada ou mesmo veículo adaptado para uma pessoa com deficiência. (MANZINI, 2005, p.82) 
A expressão Tecnologia Assistiva foi criada em 1988 como elemento jurídico dentro da legislação estadunidense para garantir o benefício de recursos e serviços favorecedores de uma vida mais independente, produtiva e incluída no contexto social (BERSCH, 2013). Portanto, o termo surge com abrangência em duas dimensões: recursos, que são os equipamentos, produtos ou sistemas, e serviços, destinados a auxiliar diretamente às pessoas com deficiência a selecionar, adquirir ou usar os recursos de TA. Entretanto, existe uma tendência equivocada em considerar como TA qualquer recurso relacionado a pessoas com deficiência, mesmo que este possa ser usado por pessoas sem deficiência com as mesmas finalidades.

Em vista ao exposto, optamos neste trabalho em definir o conceito de TA, ainda em evolução, o proposto por Teófilo Galvão Filho (2013) como

[...] um tipo de mediação instrumental, está relacionada com os processos que favorecem, compensam, potencializam ou auxiliam, também na escola, as habilidades ou funções pessoais comprometidas pela deficiência, geralmente relacionadas às funções motoras, funções visuais, funções auditivas e/ou funções comunicativas.

Nas Políticas Públicas de Ciência, Tecnologia e Inovação do Brasil têm algumas aplicações ligadas à inovação de cunho social. O Comitê de Ajudas Técnicas (CAT), instituído pela Portaria $\mathrm{N}^{\circ} 142$, de 16 de novembro de 2006, propõe o seguinte conceito para a Tecnologia Assistiva sendo

[...] uma área do conhecimento, de característica interdisciplinar, que engloba produtos, recursos, metodologias, estratégias, práticas e serviços que objetivam promover a funcionalidade, relacionada à atividade e participação de pessoas com deficiência, incapacidades ou mobilidade reduzida, visando sua autonomia, independência, qualidade de vida e inclusão social(ATA VII - Comitê de Ajudas Técnicas (CAT) - Coordenadoria Nacional para Integração da Pessoa Portadora de Deficiência (CORDE) - Secretaria Especial dos Direitos Humanos - Presidência da República).

\section{Os Núcleos de Pesquisa em Tecnologia Assistiva}

Buscando a implementação das Tecnologias Assistivas foram criados os Núcleos de Pesquisa em Tecnologia Assistiva. Essas unidades são responsáveis pela elaboração de projetos de pesquisa, desenvolvimento ou 
inovação voltados à melhoria da qualidade de vida de pessoas com deficiência ou com mobilidade reduzida. Em 2012, o orçamento liberado para a implantação dos núcleos foi de R \$ 3 milhões. Os núcleos, portanto, fazem parte de um esforço para disseminar as pesquisas de Tecnologia Assistiva para todo o território brasileiro. Este estímulo é importante porque a produção é quase artesanal, já que o equipamento deve ser adaptado a cada usuário, dificultando a produção em escala. Os núcleos atuam em rede, cuja articulação é realizada por um centro de referência, o Centro Nacional de Referência em Tecnologias Assistivas (CNRTA), localizado no Centro de Tecnologia da Informação Renato Archer (CTI), em Campinas (SP). Em janeiro de 2017 tínhamos os seguintes 52 Núcleos de Pesquisa em Tecnologia Assistiva, conforme podemos ver no quadro 1.

Quadro 1 - Núcleos de Pesquisa em Tecnologia Assistiva, 2017

\begin{tabular}{|c|l|c|}
\hline UF & \multicolumn{1}{|c|}{ INSTITUIÇÃO } & SIGLA \\
\hline AM & Instituto Federal do Estado do Amazonas & IFAM \\
\hline AM & Universidade do Estado do Amazonas & UEA \\
\hline AM & Universidade Federal do Amazonas & UFAM \\
\hline AP & Universidade Federal do Amapá & IFAP \\
\hline BA & Universidade Federal do Recôncavo da Bahia & UFRB \\
\hline CE & Instituto Federal do Ceará & IFCE \\
\hline CE & Universidade Federal do Ceará & UFC \\
\hline DF & Instituto Federal de Brasília & IFB \\
\hline DF & Universidade de Brasília & UNB \\
\hline ES & Instituto Federal do Espirito Santo & IFES \\
\hline ES & Universidade Federal do Espirito Santo & UFES \\
\hline GO & Universidade Federal de Goiás & UFG \\
\hline MG & Universidade Federal de Lavras & UFLA \\
\hline MG & Universidade Federal de Minas Gerais & UFMG \\
\hline MG & Universidade Federal São Joao Del-Rei & UFSJ \\
\hline MG & Universidade Federal de Uberlândia & UFU \\
\hline MG & Universidade de Viçosa & UFV \\
\hline MG & Universidade Federal de Alienas & UNIFAL \\
\hline MS & Universidade Católica Dona Bosco & UFGB \\
\hline MS & Universidade Federal da Grande Domados & IFPA \\
\hline PA & Instituto Federal do Para & UFG \\
\hline
\end{tabular}


Marcus Vinícius Albrecht Anversa. The regional innovative centers of assistive technology of Brazil

\begin{tabular}{|c|c|c|}
\hline UF & INSTITUIÇÃO & SIGLA \\
\hline $\mathrm{PA}$ & Universidade do Estado do Pari & UEPA \\
\hline $\mathrm{PA}$ & Universidade Federal Rural da Amazônia & UFRA \\
\hline PB & Universidade Federal da Paraíba & UFPB \\
\hline $\mathrm{PE}$ & Universidade Federal de Pernambuco & UFPE \\
\hline PR & Instituto Federal do Paraná & IFPR \\
\hline PR & Pontifícia Universidade Católica do Paraná & PUC-PR \\
\hline PR & Universidade Estadual do Norte do Paraná & UENP \\
\hline $\mathrm{PR}$ & Universidade Federal do Paraná & UFPR \\
\hline PR & Universidade Tecnológica Federal do Paraná & UTFPR \\
\hline RJ & Instituto Nacional de Tecnologia & INT \\
\hline RJ & Pontifícia Universidade Católica do Rio de Janeiro & PUC-RIO \\
\hline RJ & Universidade Federal Fluminense & UFF \\
\hline RJ & Universidade Federal do Rio De Janeiro & UFRJ \\
\hline $\mathrm{RN}$ & Universidade Federal do Rio Grande do Norte & UFRN \\
\hline RR & Universidade Federal de Roraima & UFRR \\
\hline $\mathrm{RS}$ & Centro Universitário Feevale & FEEVALE \\
\hline RS & Instituto Federal do Rio Grande do Sul & IFRS \\
\hline RS & Universidade de Caxias do Sul & UCS \\
\hline $\mathrm{RS}$ & Universidade Federal do Rio Grande do Sul & UFRGS \\
\hline $\mathrm{SC}$ & Instituto Federal de Santa Catarina & IFSC \\
\hline $\mathrm{SC}$ & Universidade Federal de Santa Catarina & UFSC \\
\hline $\mathrm{SC}$ & Centro Universitário Leonardo da Vinci & UNIASSELVI \\
\hline $\mathrm{SC}$ & Universidade do Vale do Itajaí & UNIVALI \\
\hline SE & Universidade Federal de Sergipe & UFS \\
\hline $\mathrm{SP}$ & Universidade Federal do ABC & UFABC \\
\hline $\mathrm{SP}$ & Universidade Federal de São Carlos & UFSCAR \\
\hline $\mathrm{SP}$ & Universidade Estadual Paulista Júlio de Mesquita FilHo & UNESP \\
\hline SP & Universidade Estadual de Campinas & UNICAMP \\
\hline SP & Universidade Camilo Castelo Branco & UNICASTELO \\
\hline SP & Universidade Federal de São Paulo & UNIFESP \\
\hline $\mathrm{SP}$ & Universidade de São Paulo & USP \\
\hline
\end{tabular}

Fonte: CNRTA/CTI, 2017. 


\section{Os Centros Regionais Inovativos de Tecnologia Assistiva do Brasil}

Temos como base para a definição de Centro Regional Inovativo de Tecnologias Assistiva do Brasil, embora no caso brasileiro os seus Sistemas de Inovação ainda estejam imaturos, um similar ao proposto por Marcus Anversa (2017) para os "Centros Regionais Inovativos". Este o definiu através da agregação dos correspondentes à "Milieux Inovador", do GREMI fundado por Philippe Aydalot em 1984, atual GERI (Grupo de Estudo e Pesquisa sobre Inovação), aos de Sistemas de Inovação Regionais (incluso o de Regiões de Aprendizagem²), que se originam dos Sistemas de Inovação Nacionais, com forte ligação com a Teoria da Economia Evolucionária, na quais as inovações produzidas sob essa perspectiva distinguem-se pela interação entre diversos atores, pelo caráter cumulativo do conhecimento e pela especificidade do processo de P\&D.

Temos, então, o Centro Regional Inovativo de Tecnologia Assistiva como o [Território que possui um conjunto de elementos materiais (recursos humanos, empresas, ICTs, infraestrutura), imateriais (conhecimento, fluxos informacionais) e institucionais (regras e arcabouço legal) compondo complexas redes de relações voltadas para as inovações nas quais são produzidas pela interação destes diversos atores, pelo caráter cumulativo do conhecimento e pela especificidade do processo de P\&D que acabam caracterizando a sua expertise em Tecnologia Assistiva]. Para a determinação dos Centros Regionais Inovativos de Tecnologia Assistiva neste trabalho é imprescindível a existência de NITs em funcionamento nas Instituições Científicas e Tecnológicas (ICTs) que também possuam um Núcleo de Pesquisa em Tecnologia Assistiva ligado à rede do Centro Nacional de Referência em Tecnologias Assistivas (CNRTA).

Um Núcleo de Inovação Tecnológica (NIT) é definido na Lei N. 10.973, de 02/12/2004, conhecida como Lei de Inovação Tecnológica, como sendo o núcleo ou órgão constituído por uma ou mais Instituição de Ciência e Tecnologia (ICT) com a finalidade de gerir sua política de inovação. Há diferentes modelos de NIT e dependem das especificidades de

2 Está relacionada com a ideia de região, apresentada como um sistema de aprendizagem que, progressivamente, vai sendo melhorado pelas interações entre as Empresas, as ICTs e o Governo. Dentro dessa abordagem, emerge outra, a chamada Região de Aprendizagem (Learning Region), na qual "são destacados o lugar das redes e do associativismo nos processos de desenvolvimento, em geral, e de inovação, em especial, e a ideia de que a inovação é um processo interativo, que ocorre por meio de uma diversidade de rotinas institucionais e convenções sociais" (MORGAN, 1997, apud TARTARUGA, 2014, p. 95). 
cada ICT ou consórcio de ICT e dos mecanismos de transferência de tecnologia utilizados por elas. Os NITs são a expressão baseada na visão que norteia as políticas tecnológicas realizadas em vários países com o foco em programas de cooperação entre os setores público e privado, buscando incentivar e apoiar os esforços das empresas, reduzir riscos e maximizar os resultados da capacitação científica formada localmente (LOTUFO, 2009). O autor ressaltou que esses esforços, além de estimular parcerias entre universidades, institutos de pesquisa e empresas, estão direcionados à maior interação entre as próprias empresas, seja por meio de políticas explícitas ou através de "redes cooperativas" de pesquisa, centros compartilhados e/ou infraestruturas comuns. A Lei da Inovação Tecnológica em seu Artigo 17 determina que: “A ICT deverá dispor de Núcleo de Inovação Tecnológica, próprio ou em associação com outras ICT, com a finalidade de gerir sua política de inovação" (BRASIL, 2004).

Para a determinação de cada Centro Regional Inovativo de Tecnologia Assistiva foram aferidos os seus pedidos de patentes, como demonstra o quadro 2. Na delimitação da região, a escala é a municipal e sua área de influência. Os Centros Regionais Inovativos de Tecnologia Assistiva identificados são os que possuem ICTs com NITs cadastrados no Formulário para Informações sobre a Política de Propriedade Intelectual das Instituições Científicas e Tecnológicas do Brasil (FORMICT) 2015, ano base 2014, e pelo Fórum Nacional de Gestores de Inovação e Transferência de Tecnologia (FORTEC) em 2013, além de conterem um Núcleo de Pesquisa em Tecnologia Assistiva articulado à rede do CNRTA em janeiro de 2017. O recorte temporal para a apuração dos pedidos de patentes dos referidos NITs foi de 01/01/2006 a 19/07/2017. As buscas realizadas no Banco de Dados de Dados de Patentes do Instituto Nacional da Propriedade Industrial (INPI) foi estabelecido através do uso das seguintes palavras-chaves: deficiência visual, deficiência auditiva, deficiência comunicativa, cadeirante, mobilidade e tecnologia assistiva. Todas as buscas com as devidas análises de estarem qualificadas como patentes de Tecnologia Assistivas, além de evitar a redundância dos dados.

Quanto ao uso de dados quantitativos das patentes temos que atentar na existência de algumas limitações que devem ser observadas, como bem afirmou Zvi Griliches (1990, p. 296). Primeiramente, nem toda invenção é patenteada, pois para patentear uma invenção é necessário tornar público 
os detalhes referentes a ela e consequentemente, algumas empresas preferem manter em segredo os detalhes técnicos e decidem não patentear. Outra limitação refere-se à impossibilidade de avaliar a qualidade de cada patente. Assim, mesmo não possuindo o mesmo valor econômico, elas são contadas de forma igual. $\mathrm{O}$ fato é que nem toda patente é uma inovação. Em geral, de cada 100 patentes concedidas, somente algumas se tornarão inovações, isto é, para uso no mercado. Como atesta Eduardo Albuquerque (2000), estas limitações, no entanto, não impede que as patentes sejam consideradas uma importante fonte sobre atividades tecnológicas em um sistema de inovação. Nesta ótica, a geração de pedidos de patentes de cunho tecnológico, no nosso caso, as de TA, representam um bom indicador, em termos quantitativos, o mais utilizado para aferir a produção tecnológica de dado local, além de demonstrar a prova material e cabal dos resultados de parcerias e interações entre ICTs e empresas na realização de projetos de P, D \& I.

Quadro 2. Quantidade de pedidos de patentes nos Centros Regionais Inovativos de Tecnologia Assistiva no período de 01/01/2006 a 19/07/2017

\begin{tabular}{|l|c|c|}
\hline $\begin{array}{c}\text { Centros Regionais Inovativos de } \\
\text { Tecnologia Assistiva }\end{array}$ & Pedidos de Patentes & $\begin{array}{c}\text { ICT/Quantidade de } \\
\text { Patentes }\end{array}$ \\
\hline \multicolumn{2}{|c|}{ Região Norte } \\
\hline Manaus & 1 & UFAM(l) \\
\hline Região Nordeste & 1 & IFCE (1) \\
\hline Fortaleza & 1 & UEPB (1) \\
\hline João Pessoa & 1 & UNB (1) \\
\hline \multicolumn{2}{|c|}{ Região Centro-Oeste } \\
\hline Brasília & 1 & UNIFAL(1) \\
\hline \multicolumn{2}{|c|}{ Região Sudeste } \\
\hline Alfenas & 1 & UFMG (1) \\
\hline Belo Horizonte & 1 & CTI (4) \\
\hline Campinas & 4 & UFABC (1) \\
\hline Rio de Janeiro & 3 & UFSCAR (1) \\
\hline Santo André & 1 & USP (1) \\
\hline São Carlos & 1 & UFU(1) \\
\hline São Paulo & 1 & IFES (1) \\
\hline Uberlândia & 1 & \\
\hline Vitória & 1 &
\end{tabular}




\begin{tabular}{|l|c|c|}
\hline $\begin{array}{c}\text { Centros Regionais Inovativos de } \\
\text { Tecnologia Assistiva }\end{array}$ & Pedidos de Patentes & $\begin{array}{c}\text { ICT/Quantidade de } \\
\text { Patentes }\end{array}$ \\
\hline \multicolumn{2}{|c|}{ Região Sul } \\
\hline Florianópolis & 1 & IFSC (1) \\
\hline Novo Hamburgo & 1 & FEEVALE (1) \\
\hline Porto Alegre & 1 & IFRS (1) \\
\hline
\end{tabular}

Fonte: Banco de Dados de Patentes - INPI, 2017; CTI, 2016. Organizado por Marcus Anversa, 2017.

Foram também apuradas algumas ICTs com NITs implementados que ainda não aderiram à rede do CNRTA até janeiro de 2017, mas que realizaram pedidos de patentes de Tecnologia Assistiva no período do recorte temporal da pesquisa. São elas, com o seus respectivos quantitativos: SOCIESC (1), CERTI (1), PUC-SP (1), SENAI RJ (2) e SENAI SP (2).

De acordo com o quadro 2, apresenta-se como um dos principais Centros Regionais Inovativos de Tecnologia Assistiva do Brasil o localizado em Campinas - SP, no qual se encontra o articulador da rede de Núcleos de Pesquisa em Tecnologia Assistiva, isto é, o CNRTA, localizado no Centro de Tecnologia da Informação Renato Archer (CTI). O destaque do Centro Regional Inovativo de Tecnologia Assistiva de Campinas é devido o território dispor de abundante capital de risco que o consolida em se tornar um dos maiores centros tecnológicos do país nas quais as ICTs nele localizadas tendem a produzir mais resultados a serem partilhados. De fato, este Centro se encontra no eixo econômico-tecnológico do Brasil (Rio de Janeiro-Campinas).

Na figura 1 percebe-se que os inventores independentes são os principais depositantes de patentes de tecnologia assistiva no Brasil no recorte temporal estabelecido, correspondendo à $64 \%$, seguido pelas empresas, $20 \%$ e pelas ICTs, $16 \% \%$. Foi notado que o investimento em tecnologias assistivas para a mobilidade de pessoas com deficiência não está concentrado em grandes empresas, mas sim nos inventores independentes, o que transparece que o público dessa demanda não é o foco destas empresas. Esta situação transparece a falta de comunicação/conexão entre os inventores independentes, também das ICTs, com as empresas, estas responsáveis em tornar os inventos em inovações, isto é, levar a patente ser apropriada pelo sistema produtivo. Eis um bom desafio para os NITs quanto a transferência tecnológica assistiva, assim como de programas governamentais 
como o da Associação Empresa Brasileiras de Pesquisa e Inovação Industrial (EMBRAPII).

Figura 1. Depósitos de patentes brasileiras por categoria de inventores (01/01/2016 a 19/07/2017).

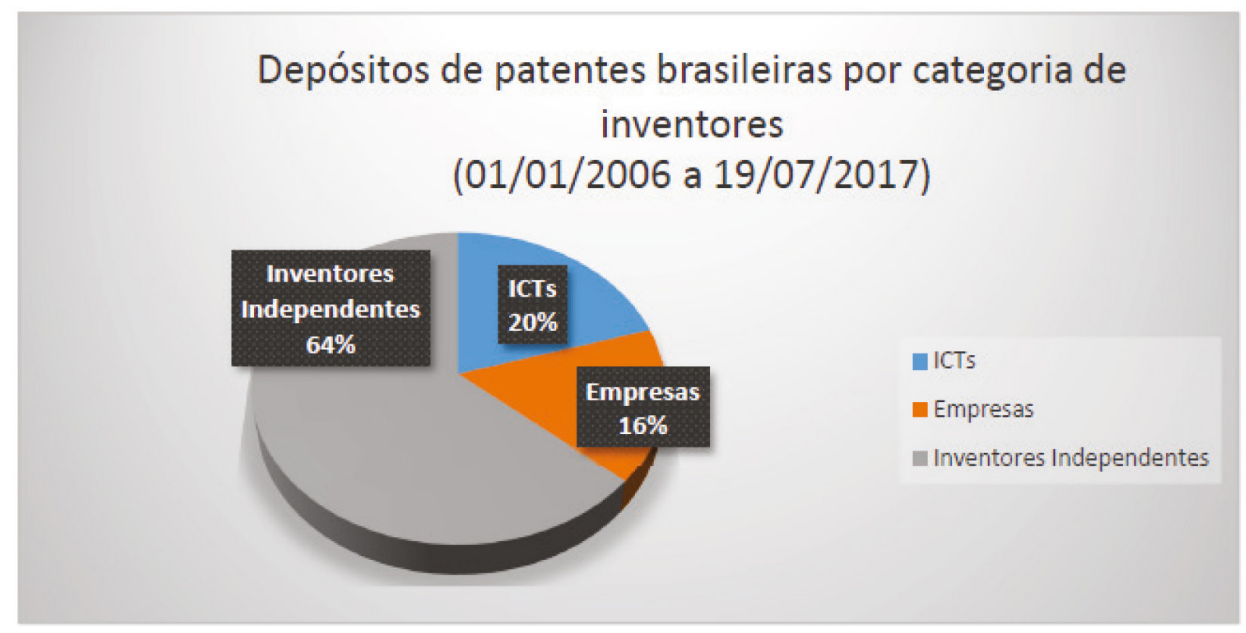

Fonte: Banco de Dados de Patentes - INPI, 2017. Organizado por Marcus Anversa, 2017.

$\mathrm{Na}$ verdade, o Brasil apresenta o gargalo de suas patentes ser apropriadas pelo sistema produtivo, caso semelhante às diversas nações, como Barbados, a melhor colocada da América Latina e Caribe no ranking do Índice Global de Inovação (GII) 2015e Costa Rica no GII 2013, além das demais nações menos desenvolvidas tecnologicamente localizadas na América Latina, África e grande parte da Ásia e Oceania.

Suponha-se existir nos citados Barbados e Costa Rica os encadeamentos produtivos ligados às atividades mais sofisticadas, que ajudariam no aumento da produtividade com a absorção e adaptação das novas patentes de potencial inovativo por parte de suas empresas locais. Entretanto, isto não corresponde à realidade (ANVERSA, 2015 \& 2017b). Outro fato é a existência da alta porcentagem das patentes serem originadas de não residentes ou serem realizados os pedidos de proteção no exterior.

$\mathrm{Na}$ prática, a proporção de pedidos nacionais e estrangeiros reflete o grau de desenvolvimento tecnológico dos países. Em economias mais desenvolvidas, o equilíbrio é maior. Um dos motivos básicos para a maior 
quantidade de pedidos serem de estrangeiros é devido as empresas brasileiras investirem pouco em P\&D. Na maior parte dos casos, empresas estrangeiras patenteiam no mercado brasileiro inovações e produtos desenvolvidos em centros tecnológicos das suas matrizes ou em outras partes do mundo. Devemos acrescentar que somado à falta de P\&D local, a quantidade de empresas estrangeiras aumentou no país na segunda metade da década de 1990 atraídas pela estabilidade econômica e aumento de consumo proporcionado com a criação do Plano Real. A criação do Mercosul também influenciou nessa atração de empresas estrangeiras.

A industrialização de países como a Argentina, México, Brasil, entre outros, se fez com forte presença de empresas estrangeiras, as quais não internalizaram seus esforços de pesquisa, desenvolvimento e inovação (P, D\&I) em suas plantas dos respectivos países, usando a América Latina como parâmetro. Isso limitou a eficácia de geração de capacitação tecnológica e de processos inovativos que assegurem aptidão para competir nos mercados globais e na integração produtiva entre os mesmos (CAMPOLINA \& DINIZ, 2013). Eis o gargalo que enfrentam as patentes brasileiras, aumentado com as relacionadas às tecnologias assistivas, em alcançarem o sistema produtivo, sendo as últimas não estarem no foco principal das grandes e médias empresas localizadas no país.

$\mathrm{Na}$ ótica dos Centros Regionais Inovativos de Tecnologia Assistiva percebe- se que o desenvolvimento econômico está intimamente ligado à capacidade interna de seus agentes, isto é, elementos materiais (empresas, ICTs, infraestrutura), imateriais (conhecimento, fluxos informacionais) e institucionais (regras e arcabouço legal), no apoio à atividade inovativa. Acrescenta-se ainda que a desigualdade existente entre as estruturas de suporte à inovação nas Macrorregiões do Brasil (divisão regional oficial do Brasil estabelecida pelo IBGE) levam a figurar como um novo mecanismo indutor da concentração socioespacial da renda, propiciando que as macrorregiões Sudeste e Sul, possuidoras de melhores condições de sustentar a inovação tecnológica, entre elas as assistivas, o desenvolvimento cada vez mais, quantitativamente e qualitativamente, de novos Centros Regionais Inovativos. Isto é obtido através de dinâmicas mais aceleradas de sua boa infraestrutura em relação às regiões Norte, Nordeste e Centro-Oeste. Por meio desta análise, constata-se que há uma expressiva associação entre variáveis ligadas ao desenvolvimento econômico e aquelas ligadas à 
estrutura tecnológica nas Macrorregiões do Brasil e seus respectivos Centros Regionais Inovativos, no nosso caso, os de Tecnologia Assistiva. Esta situação, como já exposta, ajuda a explicar o realce do Centro Regional Inovativo de Tecnologia Assistiva de Campinas, território com abundante capital de risco, em se consolidar como o maior centro tecnológico do país, nas quais as ICTs aí instaladas tendem a produzir mais resultados.

Como já notado, o presente cenário é preocupante, pois leva a uma possível perpetuação da desigualdade socioespacial, já que as regiões economicamente estruturadas e desenvolvidas tenderão a manter maior capacidade de inovar e consequentemente, maior competitividade e renda, apesar de regiões como a do Centro-Oeste estarem apresentando condições de superação deste quadro. Resultados encontrados em trabalhos comoo de Simões et al. (2005) e Marcus Anversa (2017) corroboram com a noção de que o Brasil convive ainda com um Sistema Nacional de Inovação limitado, impactando nas invenções de tecnologia assistiva, o que reflete no alto grau de concentração das indústrias empregadoras de alta tecnologia nas Regiões Sudeste e Sul. Atualmente, os editais de fomento reservam um percentual destinado apenas para os Estados das regiões menos favorecidas, justamente para tentar induzir a descentralização. Temos, então, o importante papel da ação governamental, de suas Políticas Públicas em Ciência, Tecnologia e Inovação, em atenuar tais desequilíbrios regionais.

Outro aspecto a abordar, apresentado por Maíra Corrêa (2008), é da existência de grandes dificuldades para ultrapassar as três lógicas constitutivas do perfil dos comitês gestores voltados para a inovação, na qual está incluso a social: a lógica excelentista autonomista (dos grupos hegemônicos na coletividade científica), a lógica da competitividade (empresários e gestores públicos que buscam atender aos requerimentos do mercado) e a lógica assistencial, isto é, gestores públicos que buscam resolver carências e demandas de inclusão social de forma simples e rápida sem mudanças significativas nas estruturas de educação e, mesmo, na lógica da produção e divulgação de Ciência, Tecnologia \& Inovação.

O Ministério da Ciência, Tecnologia, Inovação e Comunicações (MCTIC) possui uma Secretaria de Inclusão Social (SECIS), que a partir da gestão do ministro Gilberto Kassab em 2016, passou a ser designado Departamento de Políticas e Programas para Inclusão Social (DEPIS), com a função de promover a inclusão social por meio de ações que melhorem a 
qualidade de vida e estimulem a geração de ocupação e renda por meio do uso das Tecnologias Sociais. Até a mudança de denominação para DEPIS, era visível no site institucional do antigo MCTI, a SECIS apresentando as Tecnologias Sociais como: "Essas tecnologias caracterizam-se pela simplicidade, baixo custo e fácil aplicação, que potencializam a utilização de insumos locais e mão de obra disponível, protegem o meio ambiente, têm impacto positivo e capacidade de resolução de problemas sociais". Segundo Maíra Corrêa (2008, p. 118), "essa perspectiva, corre o risco de restringir o conceito de Tecnologias Sociais às tecnologias de segunda classe e simples instrumento assistencialista". A autora também apresenta a questão de haver a tendência de excluir do campo das Tecnologias Sociais todas aquelas tecnologias baseadas em desenvolvimentos científicos mais sofisticados e/ou que envolvam recursos de maior vulto.

Em 2017, o site institucional do MCTIC apresenta a DEPIS com algumas das seguintes competências em relação às tecnologias sociais:

XIV - contribuir para o desenvolvimento da Política Nacional de Tecnologia Social por meio da proposição de programas de desenvolvimento socioeconômico e de erradicação da pobreza e da miséria;

XV - estimular e apoiar projetos e ações no âmbito da Política Nacional da Pessoa com Deficiência que propiciem a interação de instituições de pesquisa com o setor produtivo e a formação de redes interinstitucionais para o desenvolvimento de produtos, serviços e tecnologias na área de tecnologia assistiva;

\section{Conclusão}

Ao finalizarmos este trabalho, conclui-se haver maiores debates sobre as formas que vêm assumindo as Políticas Públicas de Ciência, Tecnologia e Inovação do Brasil, nas quais envolvam as relações entre Estado, coletividade científica e sociedade, ampliando os estudos e discussões, entre eles, sobre as Tecnologias Assistivas. Uma das ações através de Políticas Públicas visando a remediação dos efeitos das desigualdades socioespaciais proporcionados pelas inovações tecnológicas seria o incremento dos Centros Regionais Inovativos, entre eles, os de Tecnologia Assistiva, ainda embrionários, como os de Fortaleza, João Pessoa e Manaus, além do fomento na constituição de novos nas Regiões Norte, Nordeste e Centro-Oeste. Esta ação proporcionaria que muitos dos inventos/patentes de 
qualificação assistiva estejam disponíveis ao sistema produtivo e às novas empresas a serem constituídas nas referidas regiões. Com isso busca-se o almejado desenvolvimento nacional socioespacial mais harmônico, contudo sem desprestigiar os centros existentes nas outras regiões. As empresas e demais instituições, enfim, obteriam a possibilidade de ampliar o seu portfólio com produtos assistivos, podendo aumentar a demanda por recursos humano locais na produção e proporcionado melhor qualidade de vida à população demandante destas tecnologias.

\section{Referencias}

ANVERSA, Marcus Vinícius Albrecht. A Geografia das Patentes e Inovações Tecnológicas com Base nos Núcleos de Inovação Tecnológica (NITs) do Brasil. Curitiba: Editora Prismas, 2017.

BRASIL. Livro Azul da $4^{\text {a }}$ Conferência Nacional de Ciência e Tecnologia e Inovação para o Desenvolvimento Sustentável. Brasília: Ministério da Ciência e Tecnologia/Centro de Gestão e Estudos Estratégicos, 2010.

LOTUFO, Roberto de Alencar. A Institucionalização de Núcleos de Inovação Tecnológica e a Experiência da Inova Unicamp. In. SANTOS, Marli Elizabeth Ritter dos; TOLEDO, Patrícia Tavares Magalhães de \& LOTUFO, Roberto de Alencar. Transferência de Tecnologia: Estratégias para a Estruturação e Gestão de Núcleos de Inovação. Campinas: Komedi, 2009.

ALBUQUERQUE, Eduardo da Motta e. Patentes Domésticas: Avaliando Estatísticas Internacionais para Localizar o Caso Brasileiro. Porto Alegre: Ensaios FEE, v. 21, n. 1, 2000.

BRASIL. Cartilha do Censo 2010 - Pessoas Com Deficiência. Brasília: Secretaria Nacional de Promoção dos Direitos da Pessoa com Deficiência - SNPD, 2012.

BRASIL. Formulário para Informações sobre a Política de Propriedade Intelectual das Instituições Científicas e Tecnológicas do Brasil (FORMICT) 2015 Ano Base 2014. Brasília: MCTI, 2015.

CAMPOLINA, Bernardo \& DINIZ, Clélio Campolina. Crise Global, Mudanças Geopolíticas e Inserção do Brasil. Brasília: Conselho de Desenvolvimento Econômico e Social - CDES, 2013. 
CORNELL UNIVERSITY; INSEAD \& WIPO (OMPI). The Global Innovation Index 2013. Geneva: World Intellectual Property Organization (WIPO/OMPI), 2014.

CORNELL UNIVERSITY; INSEAD \& WIPO (OMPI).. The Global Innovation Index 2015. Geneva: World Intellectual Property Organization (WIPO/OMPI), 2015.

CORRÊA, Maíra Baumgarten. Ciência, Tecnologia e Desenvolvimento Redes e Inovação Social. In. Parcerias Estratégicas. Brasília: Centro de Estudos Estratégicos - CGEE, n. 26, jun. 2008.

GALVÃO FILHO, Teófilo. A. A Construção do Conceito de Tecnologia Assistiva: Alguns Novos Interrogantes e Desafios. In: Revista da FACED - Entreideias: Educação, Cultura e Sociedade. Salvador: Faculdade de Educação da Universidade Federal da Bahia - FACED/ UFBA, v. 2, n. 1, p. 25-42, jan/jun. 2013.

GRILICHES, Zvi. Patent Statistics as Economic Indicators: a Survey. Pittsburgh: Journal of Economic Literature, v. 28, Dec. 1990.

MANZINI, Eduardo José. Tecnologia Assistiva para Educação: Recursos Pedagógicos Adaptados. In: Ensaios Pedagógicos: Construindo Escolas Inclusivas. Brasília: SEESP/MEC, p. 82-86, 2005.

SIMÕES, Rodrigo; OLIVEIRA, Alessandra; GITIRANA, Ayane; CUNHA, Juliana; CAMPOS, Márcia \& CRUZ, Wellington. A Geografia da Inovação: uma Metodologia de Regionalização das Informações de Gastos em P\&D no Brasil. Revista Brasileira de Inovação, Campinas, v. 4, n. 1, jan. / jun. 2005.

ANVERSA, Marcus Vinícius Albrecht. O Caso da Costa Rica e a Fiabilidade dos Índices de Competitividade e Inovação. Porto Alegre: XVI Congresso Latino-Iberoamericano de Gestão da Tecnologia (ALTEC), 2015. Disponível em: http://altec2015.nitec.co/altec/papers/633.pdf Acesso em 08 set. 2017.

ANVERSA, Marcus Vinícius Albrecht. Liderança em Inovação na América Latina e Caribe: O que Barbados têm? La Paz: XVI Encontro de Geógrafos da América Latina, 2017b. Disponível em: https://admin. egal2017.bo/static/archivos_publicos/6.docx Acesso em 08 set. 2017.

BERSCH, Rita. Introdução às Tecnologias Assistivas. Porto Alegre, 2013. Disponível em: http://www.assistiva.com.br/Introducao_Tecnologia_Assistiva.pdf Acesso em 02 jan. 2017. 
Marcus Vinícius Albrecht Anversa. Os centros regionais inovativos de tecnologia assistiva do Brasil

BRASIL. Lei n. 10.973, de 2 de dezembro de 2004. Dispõe sobre incentivos à pesquisa científica e tecnológica no ambiente produtivo e dá outras providências. Disponível em: http://www.planalto.gov.br/ ccivil_03/_ato2004-2006/2004/lei/110.973.htm Acesso em: 10 jan. 2017.

ONU. A ONU e as Pessoas com Deficiência. Nova York, 2011. Disponível em http://www.onu.org.br/a-onuem-acao/a-onu-e-as-pessoas-om-deficiencia/. Acesso em: 02 abr. 2016.

TARTARUGA, Iván Gerardo Peyré. Inovação, Território e Cooperação: Um Panorama da Geografia Econômica do Rio Grande do Sul. Tese (Doutorado em Geografia) - Programa de Pós-Graduação em Geografia, Universidade Federal do Rio grande do Sul - UFRGS, Porto Alegre, 2014. 